University of Wollongong

Research Online

Faculty of Engineering and Information

Faculty of Engineering and Information

Sciences - Papers: Part B

Sciences

2019

An alternative form to calibrate the correlated Stein-Stein option pricing model

Xinjiang He

University of Wollongong, xinjiang@uow.edu.au

Song-Ping Zhu

University of Wollongong, spz@uow.edu.au

Follow this and additional works at: https://ro.uow.edu.au/eispapers1

Part of the Engineering Commons, and the Science and Technology Studies Commons

Research Online is the open access institutional repository for the University of Wollongong. For further information contact the UOW Library: research-pubs@uow.edu.au 


\title{
An alternative form to calibrate the correlated Stein-Stein option pricing model
}

\begin{abstract}
In this paper, we present an alternative form of the correlated Stein-Stein option pricing model, which preserves the analytic tractability of the original form and also demonstrates certain advantage for model implementation in practice. We shall show, through an empirical study, that this alternative form generally outperforms the original Stein-Stein form for the selected set of data, demonstrating that it can serve as a good competitor to the original Stein-Stein form in real markets.
\end{abstract}

\section{Disciplines}

Engineering | Science and Technology Studies

\section{Publication Details}

He, X. \& Zhu, S. (2019). An alternative form to calibrate the correlated Stein-Stein option pricing model. Computational and Applied Mathematics, 38 (2), 68-1-68-21. 


\title{
An alternative form to calibrate the correlated Stein-Stein option pricing model
}

\author{
Xin-Jiang $\mathrm{HE}^{*} \quad$ Song-Ping $\mathrm{ZHU}^{\dagger}$
}

\begin{abstract}
In this paper, we present an alternative form of the correlated Stein-Stein option pricing model, which preserves the analytic tractability of the original form and also demonstrates certain advantage for model implementation in practice. We shall show, through an empirical study, that this alternative form generally outperforms the original Stein-Stein form for the selected set of data, demonstrating that it can serve as a good competitor to the original Stein-Stein form in real markets.
\end{abstract}

\section{AMS(MOS) subject classification.}

Keywords. Alternative form, Stein-Stein model, Empirical studies.

\section{Introduction}

With the rapid development of financial markets, investments on financial assets are really popular today. Financial derivatives, as one of the main kinds of financial assets, have received enormous attention, as a result of high demand for accurate pricing of them.

*Corresponding author. School of Mathematics and Applied Statistics, University of Wollongong NSW 2522, Australia (Email: xinjiang@uow.edu.au).

${ }^{\dagger}$ School of Mathematics and Applied Statistics, University of Wollongong NSW 2522, Australia. 
Among these, European options considered in this paper are one of the most significant and basic financial instruments, which can be used for hedging or speculations.

Despite the great success of B-S (Black-Scholes) model [5] for option pricing, in which asset returns are assumed to follow a log-normal distribution, and a simple and closed-form formula for European options can be obtained, it fails to reflect the phenomenon observed in the real market, such as skew [34] and leptokurtic property [35] of financial data. The constant volatility assumption, as another main disadvantage of this model, contradicts the results of empirical studies $[15,36]$, which show that the volatility varies randomly. Both of the two major unrealistic assumptions together with some other minor ones, have certainly led to mis-pricing of options with the classical B-S formula, which has thus encouraged the development of various modifications to the B-S model.

In an attempt to deal with the mis-pricing problem of the B-S model, jumps were included to model the underlying asset. Merton [33] proposed a modification by adding a Poisson jump component to the original dynamics of the B-S model. Another well-known jump-diffusion model was proposed by Kou [28] with jump sizes modeled by a double exponential distribution. The B-S model could also be further modified with Brownian motion being replaced by a pure jump process, such as the VG model suggested by Madan et al. [32] and the CGMY model proposed by Carr et al. [6].

Another popular approach to improve the behavior of the B-S model is to incorporate non-constant volatility, which can be mainly divided into two categories, i.e., local volatility and stochastic volatility models. The former was firstly introduced in $[14,16]$, assuming that the volatility is a deterministic function of the underlying price and time. However, it was pointed out by Hagan et al. [21] that "smile dynamics" are poorly captured by local volatility models, and thus the latter category of stochastic volatility models has received much more attention among researchers and market practitioners.

Pricing options under the concept of continuous-time stochastic volatility models was first carried by Johnson [26]. At its infant stage, numerical methods have to be used to 
solve option pricing problems under stochastic volatility models. For example, Johnson \& Shanno [27] and Scott [38] adopted the Monte Carlo techniques to simulate SDE (stochastic differential equation) directly while Wiggins [42] proposed a finite difference method to solve the involved PDE (partial differential equation). However, comparing with analytical solution approaches, numerical approaches are usually less preferred in practice as the computational speed of the latter is usually slower than that of the former, posing an obstacle for practical applications as model calibration is very time-consuming and the lack of analytical pricing formula can make the situation even worse. Therefore, a large number of authors have been focusing on establishing appropriate stochastic volatility models that admit analytical formula, a typical example of which is the so-called Hull-White model [24], where a power series approximation for option prices was obtained. Although this model seems to be more attractive than those derived simply through numerical methods, there were two major disadvantages in their model. One is that they assumed zero correlation between the underlying price and the volatility, which is at odds with the reality since it violates the so-called "leverage effects" that the underlying price and the volatility should be negatively correlated [2]. Another is that the volatility process also does not possess the mean-reverting property, which contradicted the fact that the stochastic process for volatility is actually mean-reverting [3].

Two of the most famous models are the Stein-Stein model [41] and the Heston model [23]. The former incorporated an Ornstein-Uhlenbeck process [18], while the latter used the CIR model to describe the volatility process. Their popularity can be mainly attributed to the existence of a closed-form solution for European option prices, which can have some obvious advantages over other models with which option prices need to be obtained numerically. In particular, closed-form solutions can not only guarantee the computational accuracy as systematical errors would always exist when numerical methods are adopted, but also reduce the time spent on many iterations involved in model calibration, a vital process that any mathematical model needs to go through before it can be used in practice. 
In this paper, we present an alternative form of the correlated Stein-Stein model [37], which preserves its analytical tractability. Although proposing a new form for a certain model makes no sense if all the model parameters could be determined analytically and precisely, the model calibration process in practice usually requires the use of an optimization algorithm, in which different forms may not be equivalent. To understand this, readers are referred to a simple example provided in [22], where it is shown that different forms of analytical solution for a certain model could yield quite different results when empirical studies are conducted. In this sense, we propose a different form of the correlated Stein-Stein model, and empirical comparison of our form and the original form is conducted based on the S\&P 500 Index and options. Our results demonstrate that under certain market conditions, our new form could provide generally better results than the original Stein-Stein form.

The rest of the paper is organized as follows. In Section 2, we will introduce the new form of the correlated Stein-Stein model with the closed-form solution for European call option. In Section 3, some preliminary empirical studies are conducted based on the S\&P 500 Index returns and options to make comparison of our new form with the original Stein-Stein form, followed by some concluding remarks given in the last section.

\section{The new form of the correlated Stein-Stein model}

In this section, the new form is proposed under the risk-neutral measure first and then we derive a closed-form pricing formula for European options.

We begin by specifying the new model of the underlying asset $S_{t}$ and the volatility $v_{t}$ as

$$
\frac{d S}{S}=r d t+v^{\alpha} d W_{t}
$$

and

$$
d v=\left[\frac{1}{2}(1-\alpha) \sigma^{2} v^{1-2 \alpha}+\lambda_{1} v^{1-\alpha}+\lambda_{2} v\right] d t+\sigma v^{1-\alpha} d B_{t}
$$


where $W_{t}$ and $B_{t}$ are Brownian motions with correlation $\rho$. We set $\sigma>0, \alpha \in(-1,1) \backslash\{0\}$, while other requirements for parameters $\alpha, \lambda_{1}$ and $\lambda_{2}$ will be given later.

\subsection{Mean-reversion}

A mean-reverting volatility model tells us that the high and low value of the volatility is temporary and it will tend to be the average over time. Thus when the mean-reverting feature is taken into account, we ignore the stochastic term in the process of volatility and only focus on the following ordinary differential equation.

$$
d v=\left[\frac{1}{2}(1-\alpha) \sigma^{2} v^{1-2 \alpha}+\lambda_{1} v^{1-\alpha}+\lambda_{2} v\right] d t .
$$

To find the long-term trend for $v_{t}$ according to (2.3), three scenarios are discussed respectively as follows.

(1) $\lambda_{2}=0$.

In this case we only need to consider $d v=\left[\frac{1}{2}(1-\alpha) \sigma^{2} v^{1-2 \alpha}+\lambda_{1} v^{1-\alpha}\right] d t$. As a result,

$$
\frac{v^{2 \alpha-1} d v}{\lambda_{1} v^{\alpha}+\frac{1}{2}(1-\alpha) \sigma^{2}}=d t
$$

Setting $u=v^{\alpha}$ in Equation (2.4) yields

$$
\frac{u d u}{\lambda_{1} u+\frac{1}{2}(1-\alpha) \sigma^{2}}=\alpha d t
$$

which implies

$$
\left[\frac{1}{\lambda_{1}}-\frac{(1-\alpha) \sigma^{2}}{\lambda_{1}} \frac{1 / 2}{\lambda_{1} u+\frac{1}{2}(1-\alpha) \sigma^{2}}\right] d u=\alpha d t .
$$

Integrating on the both sides of Equation (2.5) yields

$$
\left[\lambda_{1} u+\frac{1}{2}(1-\alpha) \sigma^{2}\right] e^{-\frac{2 \lambda_{1} u}{(1-\alpha) \sigma^{2}}}=C \cdot e^{-\frac{2 \alpha \lambda_{1}^{2}}{(1-\alpha) \sigma^{2}} t},
$$


where $C$ is a constant. Now, given the fact that the RHS (right hand side) of (2.6) is dependent on the value of $\alpha$ and there are two subsets of $\alpha$ values, $\alpha \in(-1,0) \cup(0,1)$, one needs to discuss the following two cases separately, depending on the $\alpha$ value being positive and negative, respectively.

- If $\alpha \in(-1,0)$, then

$$
\lim _{t \rightarrow \infty}\left[\lambda_{1} u+\frac{1}{2}(1-\alpha) \sigma^{2}\right] e^{-\frac{2 \lambda_{1} u}{(1-\alpha) \sigma^{2}}}=\infty
$$

from which we can obtain $\lim _{t \rightarrow \infty} u=\infty$. As a result,

$$
\lim _{t \rightarrow \infty} v=\lim _{t \rightarrow \infty} u^{\frac{1}{\alpha}}=0
$$

which contradicts the fact that $v>0$.

- If $\alpha \in(0,1)$, we have

$$
\lim _{t \rightarrow \infty}\left[\lambda_{1} u+\frac{1}{2}(1-\alpha) \sigma^{2}\right] e^{-\frac{2 \lambda_{1} u}{(1-\alpha) \sigma^{2}}}=0,
$$

from which we have

$$
\lim _{t \rightarrow \infty} v=\left[-\frac{(1-\alpha) \sigma^{2}}{2 \lambda_{1}}\right]^{\frac{1}{\alpha}}
$$

It is obvious that $\lambda_{1}$ should be negative since $v_{t}>0$.

(2) $\lambda_{2}<0$.

We set $k=\frac{\lambda_{2}}{\frac{1}{2}(1-\alpha) \sigma^{2}-\frac{\lambda_{1}^{2}}{4 \lambda_{2}}}$. Obviously $k<0$. So Equation $(2.3)$ becomes

$$
\frac{d v^{2 \alpha}}{1+k\left(v^{\alpha}+\frac{\lambda_{1}}{2 \lambda_{2}}\right)^{2}}=\frac{2 \alpha \lambda_{2}}{k} d t
$$


By applying the transform $u=v^{\alpha}$ into Equation (2.8), the following is obtained.

$$
\frac{u d u}{1+k\left(u+\frac{\lambda_{1}}{2 \lambda_{2}}\right)^{2}}=\frac{\alpha \lambda_{2}}{k} d t
$$

which can be further calculated as

$$
\left[\frac{A}{1+\sqrt{-k}\left(u+\frac{\lambda_{1}}{2 \lambda_{2}}\right)}+\frac{B}{1-\sqrt{-k}\left(u+\frac{\lambda_{1}}{2 \lambda_{2}}\right)}\right] d u=-\frac{\alpha \lambda_{2}}{k} d t
$$

where

$$
A=\frac{\lambda_{1}}{4 \lambda_{2}}+\frac{1}{2 \sqrt{-k}}, \quad B=\frac{\lambda_{1}}{4 \lambda_{2}}-\frac{1}{2 \sqrt{-k}}
$$

No matter what the value of $\lambda_{1}$ is, we can always show that $A>0, B<0$ (the proof is left in Appendix A). As a result, Equation (2.9) can be solved as

$$
\left[1+\sqrt{-k}\left(u+\frac{\lambda_{1}}{2 \lambda_{2}}\right)\right]^{A} \cdot\left[1-\sqrt{-k}\left(u+\frac{\lambda_{1}}{2 \lambda_{2}}\right)\right]^{-B}=C \cdot e^{\frac{\alpha \lambda_{2}}{\sqrt{-k}} t} .
$$

Since $\alpha$ can take either positive or negative values, we have the following two scenarios.

- If $\alpha \in(-1,0)$, then

$$
\lim _{t \rightarrow \infty}\left[1+\sqrt{-k}\left(u+\frac{\lambda_{1}}{2 \lambda_{2}}\right)\right]^{A} \cdot\left[1-\sqrt{-k}\left(u+\frac{\lambda_{1}}{2 \lambda_{2}}\right)\right]^{-B}=\infty
$$

which implies $\lim _{t \rightarrow \infty} u=\infty$. As a result,

$$
\lim _{t \rightarrow \infty} v=\lim _{t \rightarrow \infty} u^{\frac{1}{\alpha}}=0
$$

which contradicts the fact that $v>0$.

- If $\alpha \in(0,1)$, we have

$$
\lim _{t \rightarrow \infty}\left[1+\sqrt{-k}\left(u+\frac{\lambda_{1}}{2 \lambda_{2}}\right)\right]^{A} \cdot\left[1-\sqrt{-k}\left(u+\frac{\lambda_{1}}{2 \lambda_{2}}\right)\right]^{-B}=0
$$


What can be inferred from Equation (2.13) is that

$$
\lim _{t \rightarrow \infty} 1-\sqrt{-k}\left(u+\frac{\lambda_{1}}{2 \lambda_{2}}\right)=0
$$

since $1+\sqrt{-k}\left(u+\frac{\lambda_{1}}{2 \lambda_{2}}\right)>1+\sqrt{-k} \frac{\lambda_{1}}{2 \lambda_{2}}=2 A \sqrt{-k}>0$. As a result, Equation (2.14) implies that

$$
\lim _{t \rightarrow \infty} v=(-2 B)^{\frac{1}{\alpha}}
$$

(3) $\lambda_{2}>0$

In this case, it is impossible for the process $v_{t}$ to be mean-reverting; the proof is given below according to different values of $\alpha$.

- If $\alpha \in(0,1)$, then

$$
d v=v^{1-2 \alpha}\left(\lambda_{2} v^{2 \alpha}+\lambda_{1} v^{\alpha}+\frac{1}{2}(1-\alpha) \sigma^{2}\right) d t
$$

Hence there exists a positive number $p_{1}$ that $d v>0$ when $v^{\alpha}>p_{1}$, which means that $v$ will keep increasing in this case and this is no mean-reverting.

- If $\alpha \in(-1,0)$, then

$$
d v=v\left(\lambda_{2}+\lambda_{1} v^{-\alpha}+\frac{1}{2}(1-\alpha) \sigma^{2} v^{-2 \alpha}\right) d t
$$

which implies that there exists a positive number $p_{2}$ that $d v>0$ when $v^{-\alpha}>p_{2}$. In this case the process is again not a mean-reverting process.

All the discussion above leads to the following restrictions being imposed on the parameters in our form. In particular,

$$
\alpha \in(0,1),
$$




$$
\lambda_{2} \in(-\infty, 0]
$$

As for $\lambda_{1}$, there exist two scenarios that $\lambda_{1}<0$ when $\lambda_{2}=0$, and $\lambda_{1} \neq 0$ when $\lambda_{2}<0$.

After all the parameter restrictions are imposed, we are now ready to derive the pricing formula for European call options, which is presented in the next Section.

\subsection{Analytic pricing formula}

In this section the underlying asset price and the volatility are assumed to follow the dynamics introduced in Section 2.1, with which a closed-form pricing formula can be obtained.

Theorem 1 Let $U(S, v, t)$ be the call option price with $S_{t}$ and $v_{t}$ following the new dynamics proposed in Section 2. Then

$$
U(S, v, t)=S P_{1}-K e^{-r(T-t)} P_{2},
$$

where

$$
\begin{aligned}
& P_{j}=\frac{1}{2}+\frac{1}{\pi} \int_{0}^{\infty} R e\left[\frac{e^{-i \phi l n(K)} f_{j}}{i \phi}\right] d \phi, \quad f_{j}=e^{C(\tau ; \phi)+D(\tau ; \phi) v^{2 \alpha}+E(\tau ; \phi) v^{\alpha}+i \phi x}, \quad j=1,2, \\
& D(\tau ; \phi)=\frac{d-2 \alpha\left(b_{j}+\lambda_{2}+i \phi \rho \sigma\right)}{4 \alpha^{2} \sigma^{2}}\left(\frac{1-e^{d \tau}}{1-g e^{d \tau}}\right), \\
& E(\tau ; \phi)=\frac{\lambda_{1}\left[d-2 \alpha\left(b_{j}+\lambda_{2}+i \phi \rho \sigma\right)\right]}{2 \alpha \sigma^{2} d\left(1-g e^{d \tau}\right)}\left(4 e^{\frac{1}{2} d \tau}-2 e^{d \tau}-2\right), \\
& C(\tau ; \phi)=w(\tau ; \phi)-w(0 ; \phi)+r i \phi \tau+\frac{1}{4} q(\tau), \\
& w(t ; \phi)=\frac{\lambda_{1}^{2}\left[d-2 \alpha\left(b_{j}+\lambda_{2}+i \phi \rho \sigma\right)\right]}{2 \sigma^{2} d^{3}}\left\{\frac{4 a r c t a n\left(i \sqrt{g} e^{\frac{d t}{2}}\right) h}{i g \sqrt{g}}+\left[-2 \alpha\left(b_{j}+\lambda_{2}+i \phi \rho \sigma\right)-d\right] t d\right. \\
& \left.\quad+\frac{\left[2 \alpha\left(b_{j}+\lambda_{2}+i \phi \rho \sigma\right)-d\right]\left(6 g-4 g e^{\frac{1}{2} d t}-4 g^{2} e^{\frac{1}{2} d t}+g^{2}+1\right)}{g^{2}\left(g e^{d t}-1\right)}+\frac{(g+1) h \ln \left(g e^{d t}-1\right)}{g^{2}}\right\}, \\
& d=2 \alpha \sqrt{\left(b_{j}+\lambda_{2}+i \phi \rho \sigma\right)^{2}-\sigma^{2}\left(2 a_{j} i \phi-\phi^{2}\right),} g=\frac{2 \alpha\left(b_{j}+\lambda_{2}+i \phi \rho \sigma\right)-d}{2 \alpha\left(b_{j}+\lambda_{2}+i \phi \rho \sigma\right)+d}, \\
& h=d(1+g)-2 \alpha\left(b_{j}+\lambda_{2}+i \phi \rho \sigma\right)+2 \alpha g\left(b_{j}+\lambda_{2}+i \phi \rho \sigma\right), \\
& q(t)=\left[d-2 \alpha\left(b_{j}+\lambda_{2}+i \phi \rho \sigma\right)\right] t-2 \ln \left(\frac{1-g e^{d t}}{1-g}\right), \quad p(t)=\alpha\left(b_{j}+\lambda_{2}+i \phi \rho \sigma\right) t+\frac{1}{2} q(t),
\end{aligned}
$$




$$
a_{1}=\frac{1}{2}, a_{2}=-\frac{1}{2}, b_{1}=\rho \sigma, b_{2}=0
$$

The proof of this proposition is left in Appendix B.

Although our form would degenerate to the original version

$$
\begin{aligned}
\frac{d S}{S} & =r d t+\bar{v} d W_{t} \\
d \bar{v} & =k(\theta-\bar{v}) d t+\bar{\sigma} d B_{t}
\end{aligned}
$$

if we make the transformation of $\bar{v}=v^{\alpha}, k=-\alpha \lambda_{2}, \theta=-\frac{\lambda_{1}}{\lambda_{2} k}$ and $\bar{\sigma}=\alpha \sigma$, our results are still meaningful since different forms of a certain model could yield different results in the model calibration process due to its complicatedness, as stated in [22]. In order to further demostrate this point, the behavior of our form in real markets will be compared with the original Stein-Stein form through a carefully designed empirical study in the next section.

\section{Empirical studies}

In this section, empirical results are presented and discussed with the the original SteinStein form being taken as a benchmark to assess the performance of our form in real markets. In the following, we will firstly describe the data we use and introduce the method adopted for parameter estimation. Then, the performance of these two models is quantitatively compared by the pricing errors measured with the "distance" between model-produced and market prices, and it is widely accepted that a model is regarded as the better one if it exhibits less pricing errors. In fact, pricing errors usually consist of two parts: the so-called in-sample errors and out-of-sample errors. Conventionally, the period in which market data is available is divided into two; the first one refers to a period in which

data is used for parameter determination (referred to as the "in-sample observations") and the second one then refers to the period in which data is used to verify the performance 
of a model through a comparison between the observed option prices and the calculated option prices based on the parameters determined from the "in-sample" period (in contrast to the first period, data in the second period is usually referred to as the "out-of-sample observations"). It should be remarked that it is usually difficult to achieve lower in- and out-of-sample errors simultaneously. When comparing two models through a comparison of the in- and out-of-sample errors, it is hard to draw a conclusion if only one part of errors is less than the other. However, there should be no doubt in one's mind which model is superior if both of its in- and out-of-sample fitness are better. This is the principle based on which we draw the conclusions of our empirical studies in the subsection 3.3.

\subsection{Data description}

Our empirical study is conducted on a data set of the S\&P 500 Index and European call options written on the S\&P 500 Index from Sept 2010 to Aug 2012. However, raw data should not be adopted directly in the estimation since sample noise needs to be eliminated. Hence, several appropriate filters presented below were applied to the raw data, before they were used to estimate model parameters.

First of all, mid-prices, which equal to the average value of bid and ask prices, are used as option prices. It is well-known that wide bid-ask spread would discourage investors from trading. Thus, those samples with too much relative difference between offer and bid prices, say $\frac{\text { offer }- \text { bid }}{\text { bid }} \geq 0.05$, are removed. Secondly, following a number of authors, such as Bakshi et. al [2] and Christoffersen et. al [9], only Wednesday and Thursday options data is adopted. In particular, Wednesday options data is used in estimation since Wednesday is least likely to be a holiday in a week and also less likely to be affected by the "day-of-the-week" effect than other days such as Monday and Friday, whereas the corresponding Thursday data serves as the market price to be compared with the predicted price calculated by the estimated parameters. Another motivation for only using Wednesday data in parameter estimation is that global optimization problems are usually 
Table 1: Number of observations

\begin{tabular}{|c|c|c|}
\hline & Total & Daily average \\
\hline Original data & 543197 & 743 \\
\hline Filtered Wednesday data & 3442 & 34 \\
\hline Filtered Thursday data & 4070 & 41 \\
\hline
\end{tabular}

quite time-consuming and choosing one day a week allows us to study a relatively longer time series yielding more reliable results. Thirdly, options with less than 30 days and more than 120 days to maturity are removed since there are less time values and less information about the future dynamics of the firm [29] for options with short time to expiry, and options with long time to expiry are traded at a high premium. Fourthly, very deep in-the-money and very deep out-of-money options are discarded due to their inactivity in the market and may also have liquidity-related biases [40]. Specifically, options with the absolute moneyness, defined as the relative difference between the S\&P 500 Index value and the corresponding strike price (Moneyness $=\frac{S-K}{K}$ ) over $10 \%$ are excluded. Fifthly, options with prices less than $1 / 8$ are all removed since these prices are rather volatile [13] and such abnormal volatility may result in unusual option prices. Finally, with dividend rate assumed to be zero, options not satisfying the general arbitrage restriction, i.e. $C \geq \max (0, S-K)$, are discarded.

It should be noted that after applying the above six filters, the daily average observations on Wednesday are over 30. Thus, we have also removed those days with only less than 5 sets of option data left to avoid biases when we analyze final results. This is because our estimation is conducted daily, and with a too-small number of samples (say, less than 5 sets of daily options data), we may end up obtaining better in-sample fitness at the expense of severely losing out-of-sample fitness. In Table 2, the numbers of observations for original data and filtered data are presented, respectively. It should be noted that after these filters are applied, more efficiency can be achieved in the process of parameter estimation with all the important information still preserved according to various previously conducted empirical studies in the literature $[2,9]$. 
As for the risk-free interest rate, we chose the three-month U.S. Treasury Bill Rate, which is released daily, as a proxy of the risk-free rate $[4,40]$ since the time to expiry of the selected options is less than 120 days. Upon the preparation of all the data described above, parameter estimation was conducted with a genetic algorithm as the main tool of optimization, the details of which are described in the next subsection.

\subsection{Parameter estimation}

In this section, we first provide a brief summary of the model parameters that need to be determined in the original Stein-Stein form and then introduce a genetic algorithm used to determine model parameters.

Recall that the correlated Stein-Stein model is specified as

$$
\begin{aligned}
\frac{d S}{S} & =r d t+v d W_{t} \\
d v & =k(\theta-v) d t+\sigma d B_{t}
\end{aligned}
$$

where the following five parameters, i.e. the mean-reversion speed $k$, the long-term mean $\theta$, the volatility of volatility $\sigma$, correlation $\rho$ between $W_{t}$ and $B_{t}$ and the initial value of volatility $v_{0}$, need to be determined from real market data.

A common approach to determine model parameters is to find the set of "optimal" parameters that minimizes the "distance" between market and model prices. Actually, there are different kinds of definition for the distance, a common one is to take the percentage mean squared error (PMSE)

$$
P M S E=\frac{1}{N} \sum_{i=1}^{N}\left[\frac{C^{\text {Market }}-C^{\text {Model }}}{C^{\text {Model }}}\right]^{2}
$$

as the objective distance function to measure the relative difference between market and model prices. Here $C^{\text {Market }}$ denotes the market price of an option contract from one 
sample, $C^{\text {Model }}$ represents the corresponding calculated price with our pricing formula with a particular set of parameters and $N$ is the total number of observations selected in a single estimation. However, the main disadvantage in choosing such an objective function is that a cheap option (i.e., low $C^{\text {Market }}$ ) could place an abnormally high amount of weight in PMSE. Therefore, following Christoffersen \& Jacobs [8] and Lim \& Zhi [31], we instead chose the dollar mean-squared errors

$$
M S E=\frac{1}{N} \sum_{i=1}^{N}\left[C^{\text {Market }}-C^{\text {Model }}\right]^{2},
$$

as the objective function.

Another issue is how to choose an appropriate optimization method. It should be noticed that although local minimization, which requires an initial guess that is very close to the true optimal solution, can be much less time consuming, its results are usually not reliable since it depends too much on the selected initial guess. In fact, the objective function (3.1) is not necessarily convex and thus there could exist several local minima, which would probably result in a local minimum being taken as the global minimum. In contrast, a properly designed global optimization should able to skip local minima and correctly identify the global minimum in an efficient way.

A genetic algorithm [11] is such a global optimization with some very nice properties. It is based on the idea of natural selection, introducing stochastic factors when searching for a satisfactory result in order to skip over local optima. One of the most important reasons for us to adopt this particular algorithm is that it randomly selects a number of different initial guesses to explore the entire solution space. Moreover, a so-called "mutation" step in the optimization process allows the algorithm to avoid local minima by preventing parameters from being restricted to a small region. In the literature, genetic algorithms have been applied in finance by quite a few researchers already. For instance, Gimeno \& Nave [19] conducted estimation of the term structure of interest rates with a genetic algorithm while 
Grace [20] and Cont \& Ben Hamida [12] have adopted it in the area of option pricing. It is even pointed out by Bajpai \& Kumar [1] that genetic algorithms are one of the best global optimization methods and can provide high quality solutions since they are intrinsically parallel and can explore the solution space in multiple directions at the same time.

It should be remarked that another advantage of adopting a genetic algorithm is its implementation in the Matlab is straightforward by using a built-in function $g a$. As a result of the numerical implementation, Table 2 exhibits the estimated daily parameters extracted from the selected market data for the two models under consideration in this paper, respectively.

Table 2: Estimated parameters

\begin{tabular}{|c|c|c|c|c|c|c|c|c|}
\hline parameters & $\sigma$ & $\rho$ & $v_{0}$ & $\alpha$ & $\lambda_{1}$ & $\lambda_{2}$ & $k$ & $\theta$ \\
\hline Our form & 1.4233 & -0.5186 & 0.0363 & 0.4278 & -2.1002 & -12.3563 & & \\
\hline Stein-Stein form & 1.5710 & -0.8170 & 0.0527 & & & & 64.7818 & 0.0461 \\
\hline
\end{tabular}

A careful analysis of the extracted parameters has led to some very interesting observations/remarks on the reasonableness of the obtained parameter values. First of all, the long-term mean in the original Stein-Stein form, denoted by the parameter $\theta$, is 0.0461 , while that for our form is 0.0510 , which are quite similar to each other. In addition, our results are in the reasonable region since both of these values are also very close to many other results using different sets of data in the literature (e.g., see $[2,10])$.

Secondly, the extracted volatility of volatility in our form is 1.4233 , which is slightly smaller than that of the original Stein-Stein form. Although these results are larger than those reported in $[7,17]$, they are obviously smaller than those obtained in [30], which are over 2. Therefore, our results in this category are also in line with the existing literature and thus we believe that they are reasonable and acceptable.

Moreover, the values of correlation $\rho$ (also known as "leverage effect") for our form and the original Stein-Stein form are -0.5186 and -0.8170 respectively. Although it seems that the results are quite distinct with each other, our results are still reasonable since the 
value for correlation could vary in a large range with different sets of data. For example, the value of $\rho$ was documented to be in the range of -0.39 in [25] and -0.40 in [17]. In contrast, the results in [7] showed that it could be more negatively correlated as much as $\rho=-0.8519$.

An further check of the reasonableness of determined parameters is of course to conduct empirical comparison of the performance of our form with that of the original Stein-Stein form using these parameters, which is presented in the next subsection.

\subsection{Empirical comparison}

In this subsection, the performance of our form and the original Stein-Stein form in real markets is compared using the extracted parameters. To firstly give an idea of how both forms perform in the parameter estimation stage, the recovered options prices from the two forms are compared against the corresponding market option prices listed on two different days across all different strikes and expiry dates.

Figures 1 and 2 display the closeness between recovered prices of both forms and market prices listed on 5 Oct., 2011, with 45 and 73 days to expiry, respectively. One can clearly observe from Figure 1(a) and 2(a) that our price is generally closer to the market price for different strikes. To further demonstrate the performance of both forms, the relative errors between the recovered prices and market prices are shown in Figure 1(b) and 2(b). For the options with 45 days to expiry, the relative errors produced by our form are all lower than those produced by the original Stein-Stein form except for the two options that are very deep out-of-money, as shown in Figure 1(b). On the other hand, as far as options with 73 days to expiry are concerned, our errors shown in Figure 2(b) are all smaller than the Stein-Stein ones, demonstrating the real advantage of using the new form for this case.

Depicted in Figures 3 and 4 are the recovery results on another date, i.e., 29 Aug., 2012. Similar to what has been presented in Figures 1 and 2, the level of the relative 


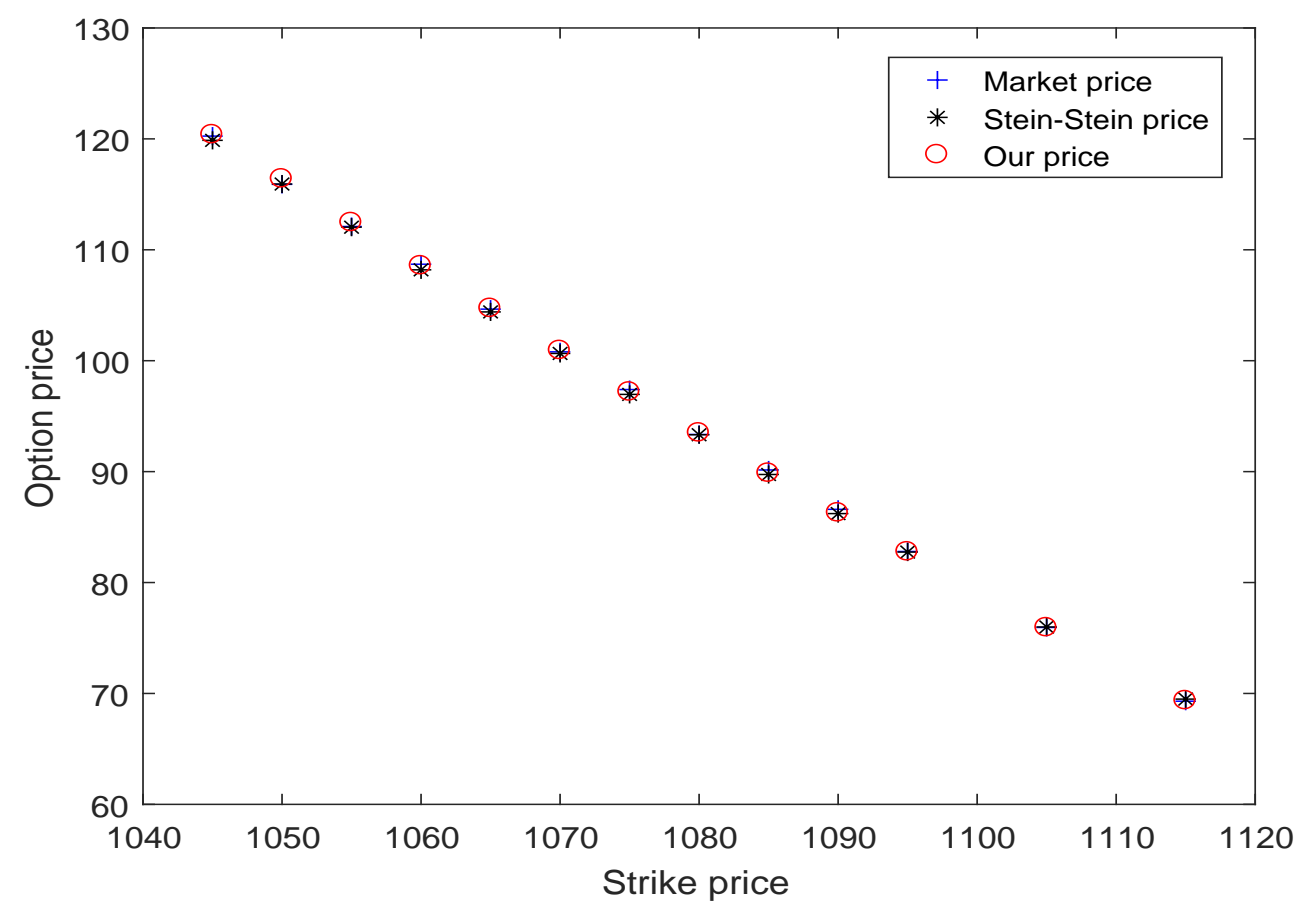

(a) Recovered prices of both forms vs market prices.

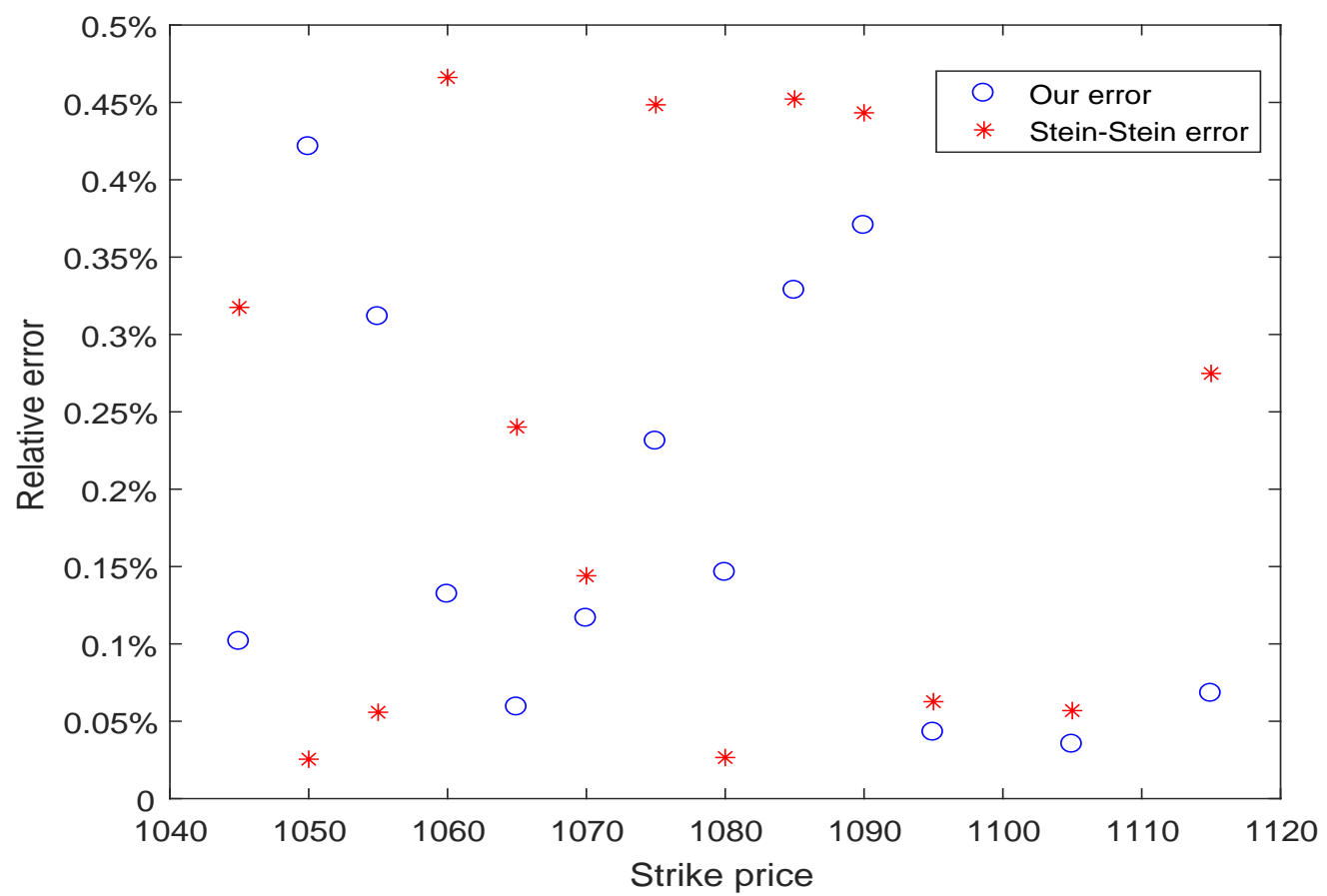

(b) The relative error between recovered prices of both forms and market prices.

Figure 1: The comparison of recovered prices of both forms and market prices on 5 Oct., 2011 with 45 days to expiry. 


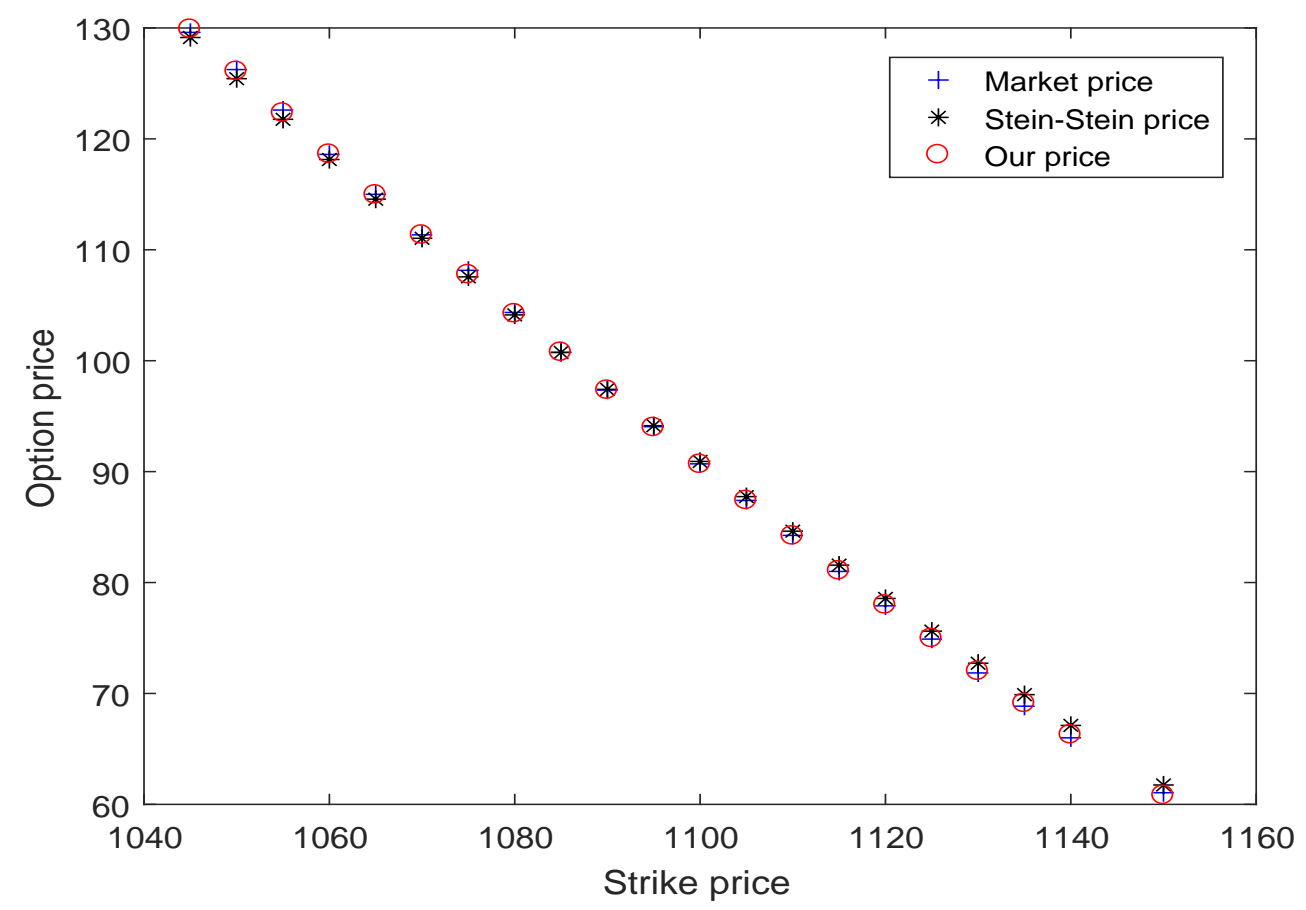

(a) Recovered prices of both forms vs market prices.

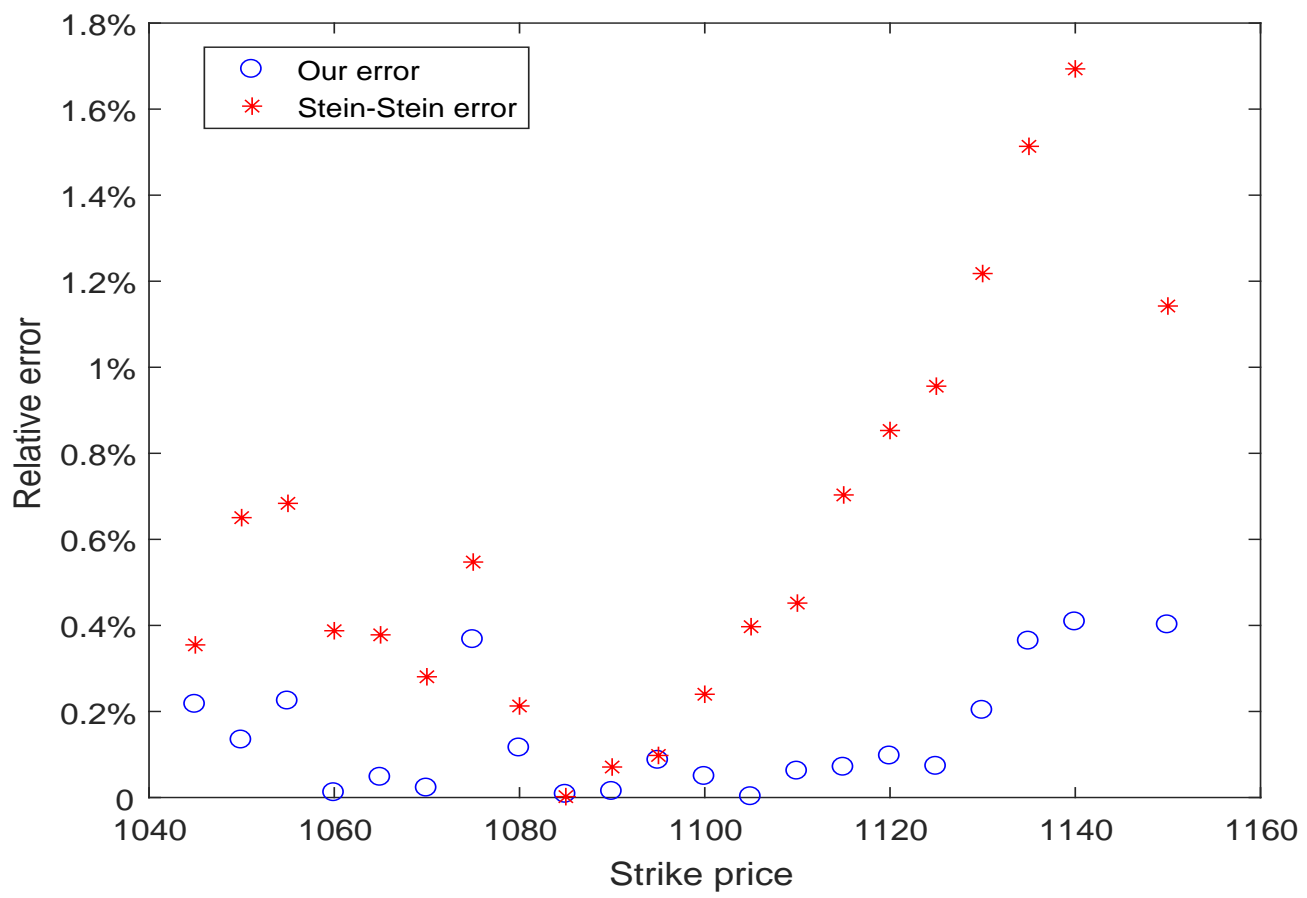

(b) The relative error between recovered prices of both forms and market prices.

Figure 2: The comparison of recovered prices of both forms and market prices on 5 Oct., 2011 with 73 days to expiry. 


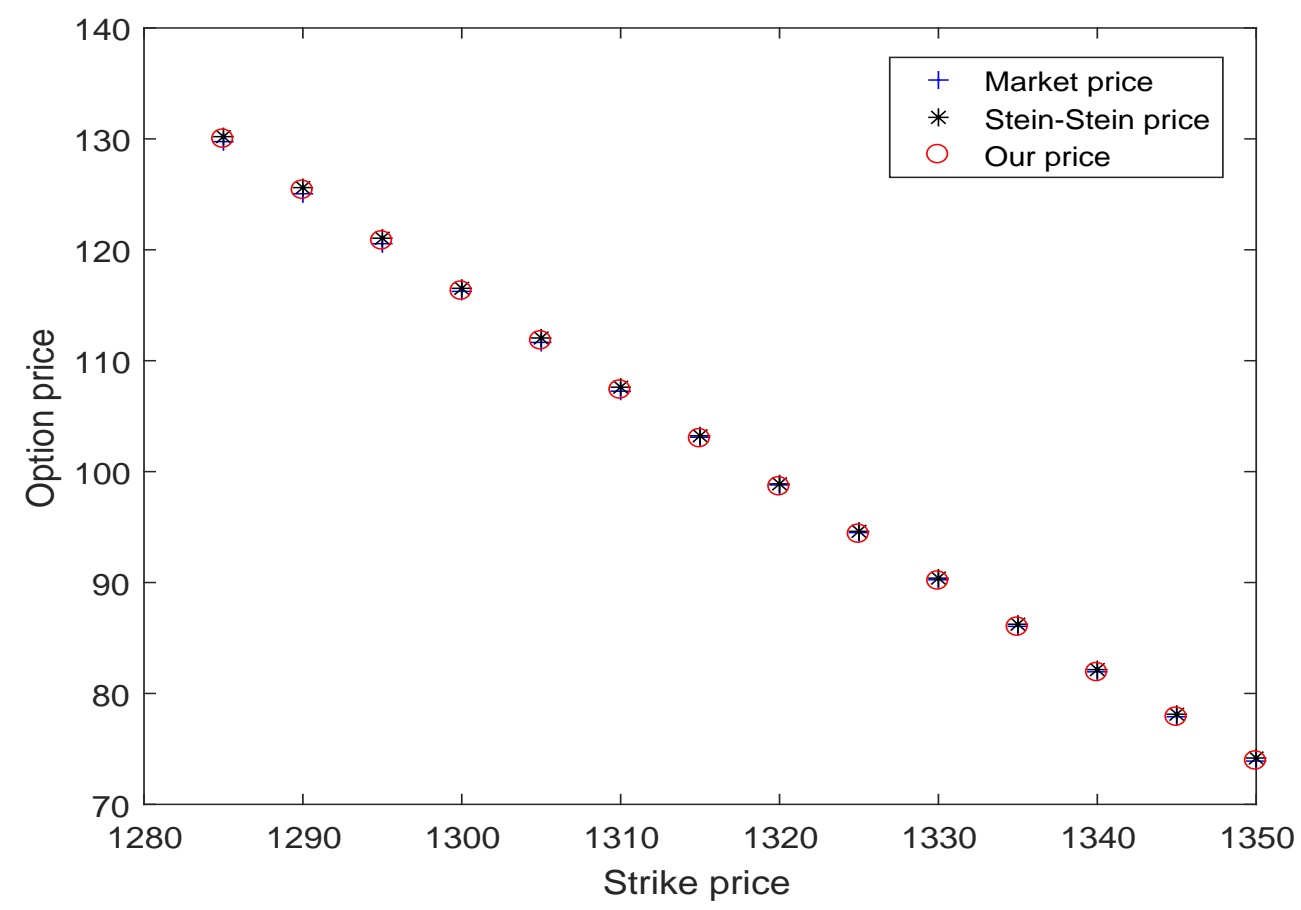

(a) Recovered prices of both forms vs market prices.

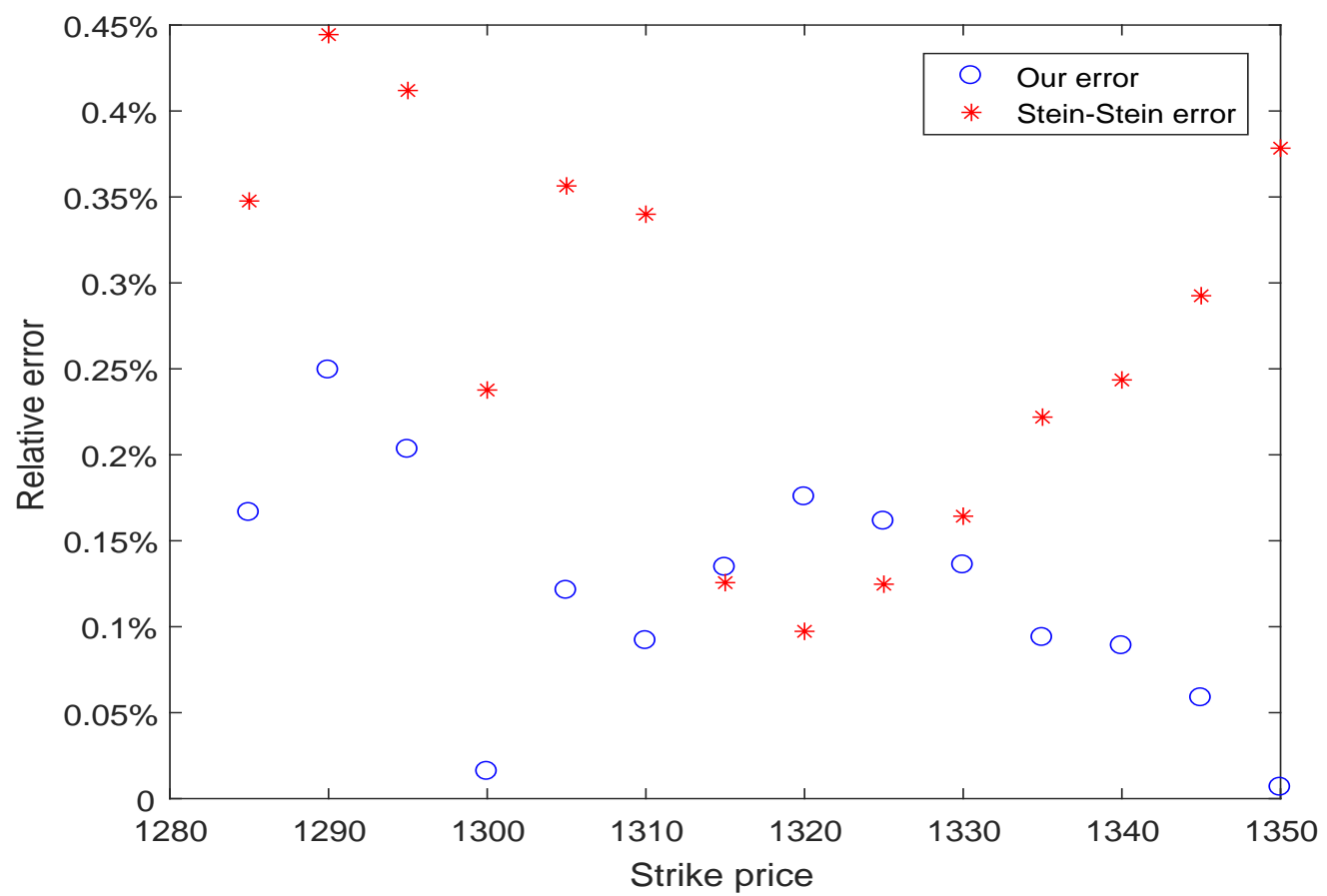

(b) The relative error between recovered prices of both forms and market prices.

Figure 3: The comparison of recovered prices of both forms and market prices on 29 Aug., 2012 with 52 days to expiry. 


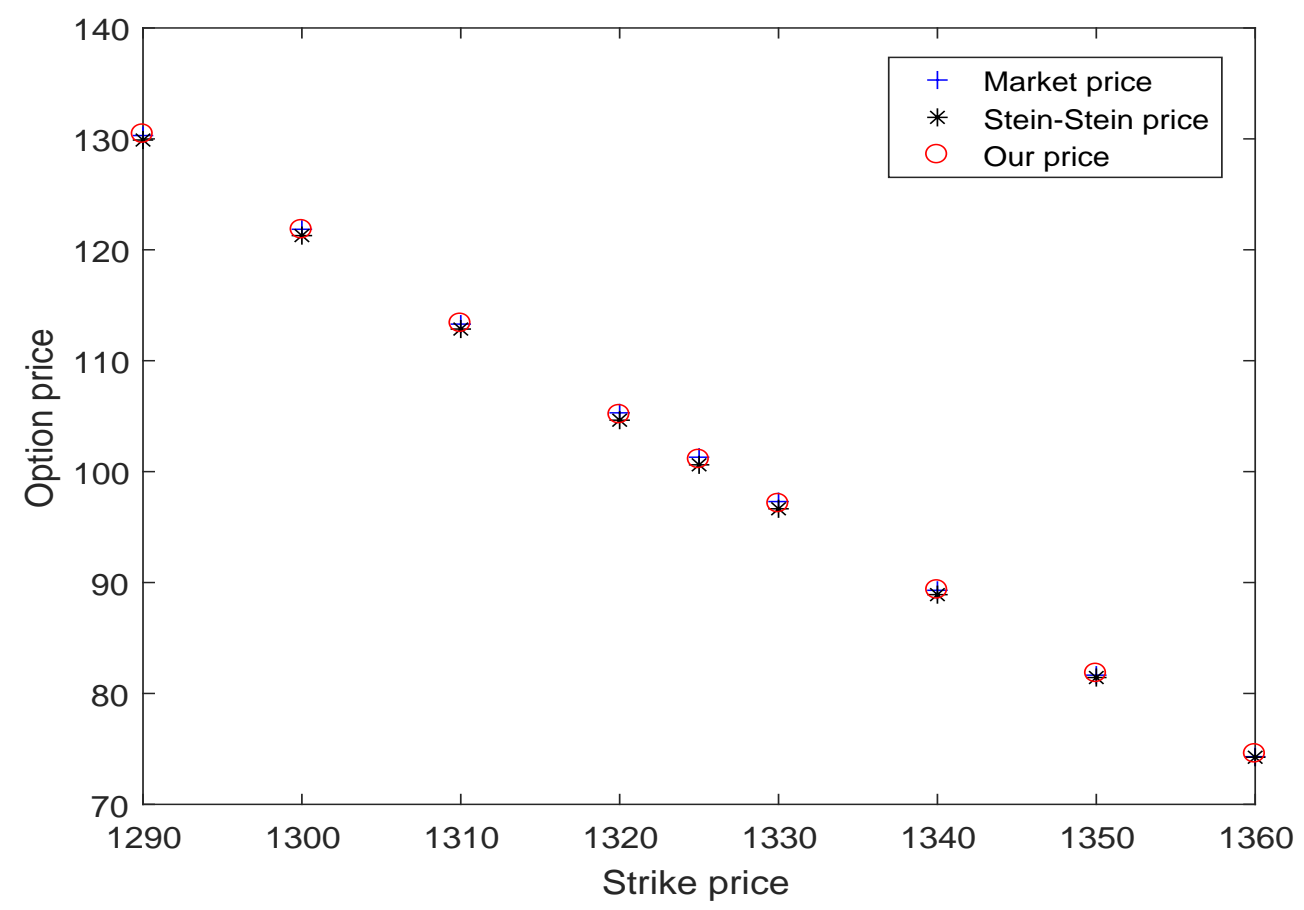

(a) Recovered prices of both forms vs market prices.

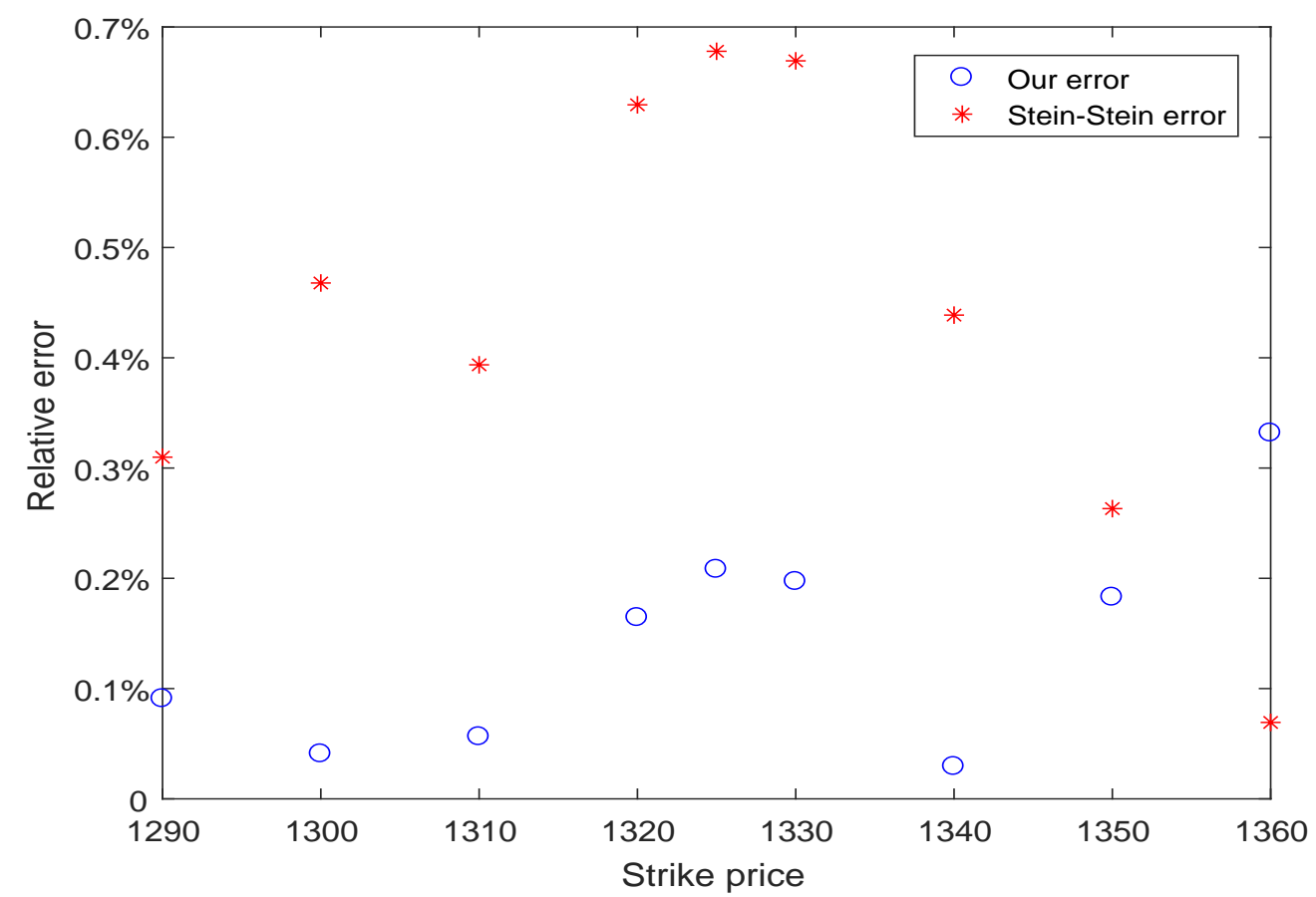

(b) The relative error between recovered prices of both forms and market prices.

Figure 4: The comparison of recovered prices of both forms and market prices on 29 Aug., 2012 with 80 days to expiry. 
error for our form is generally lower than that for the original Stein-Stein form. The only difference here is that instead of very deep out-of-money options, our form performs worse than the original Stein-Stein form when options with 52 and 80 days to expiry are close to at-the-money and in-the-money, respectively.

To summarize the model calibration results, we adopt the root mean-squared error (RMSE), which is the square root of the objective function (MSE), as a measure of "goodness of fit", since it is widely accepted that a model is regarded better if there exist less accumulated pricing errors calculated by the distance between model prices and market prices. Table 3 exhibits the in- and out-of-sample errors for the two models.

Table 3: In- and out-of-sample errors for the two forms

\begin{tabular}{|c|c|c|}
\hline Error & In-sample & out-of-sample \\
\hline Our form & 0.2806 & 1.7558 \\
Stein-Stein form & 0.3715 & 1.8309 \\
Relative difference & $22.80 \%$ & $4.10 \%$ \\
\hline
\end{tabular}

It is obvious from Table 3 that our form generally outperforms the original SteinStein form in terms of both in- and out-of-sample errors. To be more specific, from the perspective of in-sample errors, the daily averaged RMSE for our form is only 0.2806, compared with 0.3715 for the original Stein-Stein form. It is clear that our form is superior

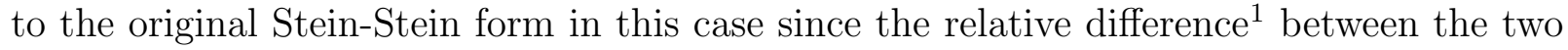
models, as far as the daily averaged RMSE is concerned, is almost 25\%, which is quite significant. On the other hand, when out-of-sample errors are taken into consideration, a similar pattern emerges; our form still shows a better performance than the original SteinStein form, although the relative difference between them has narrowed down to $4.10 \%$. Therefore, combining both in-sample and out-of-sample observations, we can conclude that our form serves as a better choice than the original Stein-Stein form for the data set chosen

\footnotetext{
${ }^{1}$ The relative difference is defined as

$$
\text { Relative difference }=\frac{\mid \text { Our form error }- \text { Stein-Stein form error } \mid}{\text { Stein-Stein form error }} .
$$
}


in this comparison.

It is also interesting to notice that the RMSE for out-of-sample errors is always much larger than that for in-sample errors. This is not difficult to understand since model prices are calculated with determined parameters; those obtained with in-sample data are believed to be "closest" to market prices of the corresponding option contracts, which implies that in-sample errors should be relatively low, while out-of-sample data are only used as the verification of option prices and certainly there is no guarantee that out-of-sample errors be low.

On the other hand, options are traded with a wide range of strikes in real markets and thus it is important to check the out-of-sample valuation errors sorted by moneyness, which are shown in Table 4. While the range of moneyness is indicated on the top row of the table, the abbreviation in the parentheses indicate "out of money" "at the money" and "in the money", respectively, from the left to the right columns.

Table 4: Out-of-sample errors according to moneyness

\begin{tabular}{|c|c|c|c|}
\hline Moneyness & $0.90<S / K<0.97(O)$ & $0.97 \leq S / K \leq 1.03(A)$ & $1.03<S / K<1.10(I)$ \\
\hline Our form & 0.9363 & 2.0645 & 1.6968 \\
Stein-Stein form & 0.9216 & 2.2155 & 1.7509 \\
Relative difference & $1.60 \%$ & $6.82 \%$ & $3.09 \%$ \\
\hline
\end{tabular}

From this table, we can see that although the original Stein-Stein form is a better choice as far as out-of-money option prices are concerned, the performance of our form is better than that of the original Stein-Stein form in the next two categories. However, the worse-off part is only $1.60 \%$ whereas the better-off parts are roughly at least doubled this percentage. Thereby, we can confidently conclude that our form has given an overall better performance than that of the original Stein-Stein form, particularly given that at or in the money performance of a model is far more important than its out of money performance. Of course, this conclusion is based on the empirical test of one set of data. It is quite possible that the performance of these two models may reverse with some other data sets. However, our empirical study presented here can at least suggest it may offer as a good 
competitor of the original Stein-Stein form for some other markets, such as commodity and futures exchange markets.

\section{Conclusion}

In this paper, an alternative form of the correlated Stein-Stein model is proposed for option pricing and model calibration. Knowing that different forms of a certain model may yield different results in the model calibration process, our form is empirically compared with the well-known original Stein-Stein form with S\& P 500 returns and options. Results show that our form generally outperforms the original Stein-Stein form, and thus it could be used as an alternative to the original Stein-Stein form for some markets.

\section{References}

[1] P. Bajpai and M. Kumar. Genetic algorithm-an approach to solve global optimization problems. Indian Journal of Computer Science and Engineering, 1(3):199-206, 2010.

[2] G. Bakshi, C. Cao, and Z. Chen. Empirical performance of alternative option pricing models. The Journal of Finance, 52(5):2003-2049, 1997.

[3] S. Beckers. Variances of security price returns based on high, low, and closing prices. Journal of Business, pages 97-112, 1983.

[4] M. A. Benjamin, H. O. Hinnant, T. T. Shigeno, and D. N. Olmstead. Multi-sensor fusion, Oct. 16 2007. US Patent 7,283,904.

[5] F. Black and M. Scholes. The pricing of options and corporate liabilities. The Journal of Political Economy, pages 637-654, 1973.

[6] P. Carr, H. Geman, D. B. Madan, and M. Yor. The fine structure of asset returns: An empirical investigation. The Journal of Business, 75(2):305-333, 2002. 
[7] P. Christoffersen, S. Heston, and K. Jacobs. The shape and term structure of the index option smirk: Why multifactor stochastic volatility models work so well. Management Science, 55(12):1914-1932, 2009.

[8] P. Christoffersen and K. Jacobs. Which garch model for option valuation? Management science, 50(9):1204-1221, 2004.

[9] P. Christoffersen, K. Jacobs, and K. Mimouni. An empirical comparison of affine and non-affine models for equity index options. Manuscript, Springer, 2006.

[10] P. Christoffersen, K. Jacobs, and K. Mimouni. Models for s\&p 500 dynamics: evidence from realized volatility, daily returns, and option prices. CREATES Research Paper, (2007-37), 2007.

[11] D. A. Coley. An introduction to genetic algorithms for scientists and engineers, volume 1655. World scientific Singapore, 1999.

[12] R. Cont and S. Ben Hamida. Recovering volatility from option prices by evolutionary optimization. 2004.

[13] A. David and P. Veronesi. Option prices with uncertain fundamentals. Olin School of Business Working Paper, pages 07-001, 2002.

[14] E. Derman and I. Kani. Riding on a smile. Risk, 7(2):32-39, 1994.

[15] B. Dumas, J. Fleming, and R. E. Whaley. Implied volatility functions: Empirical tests. The Journal of Finance, 53(6):2059-2106, 1998.

[16] B. Dupire et al. Pricing with a smile. Risk, 7(1):18-20, 1994.

[17] B. Eraker. Do stock prices and volatility jump? reconciling evidence from spot and option prices. The Journal of Finance, 59(3):1367-1404, 2004. 
[18] C. Gardiner. Stochastic methods. Springer-Verlag, Berlin-Heidelberg-New YorkTokyo, 1985.

[19] R. Gimeno and J. M. Nave. A genetic algorithm estimation of the term structure of interest rates. Computational Statistics \&3 Data Analysis, 53(6):2236-2250, 2009.

[20] B. K. Grace. Black-scholes option pricing via genetic algorithms. Applied Economics Letters, 7(2):129-132, 2000.

[21] P. S. Hagan, D. Kumar, A. S. Lesniewski, and D. E. Woodward. Managing smile risk. The Best of Wilmott, 1:249-296, 2002.

[22] X.-J. He and S.-P. Zhu. An alternative form used to calibrate the heston option pricing model. Computers \&3 Mathematics with Applications, 71(9):1831-1842, 2016.

[23] S. L. Heston. A closed-form solution for options with stochastic volatility with applications to bond and currency options. Review of Financial Studies, 6(2):327-343, 1993.

[24] J. Hull and A. White. The pricing of options on assets with stochastic volatilities. The Journal of Finance, 42(2):281-300, 1987.

[25] E. Jacquier, N. G. Polson, and P. E. Rossi. Bayesian analysis of stochastic volatility models with fat-tails and correlated errors. Journal of Econometrics, 122(1):185-212, 2004.

[26] H. Johnson. Option pricing when the variance rate is changing. Working paper, University of California, Los Angeles, 1979.

[27] H. Johnson and D. Shanno. Option pricing when the variance is changing. Journal of Financial and Quantitative Analysis, 22(02):143-151, 1987.

[28] S. G. Kou. A jump-diffusion model for option pricing. Management Science, 48(8):1086-1101, 2002. 
[29] A. Le. Separating the components of default risk: A derivative-based approach. The Quarterly Journal of Finance, 2014.

[30] S. Levendorskii. Efficient pricing and reliable calibration in the heston model. International Journal of Theoretical and Applied Finance, 15(07):1250050, 2012.

[31] K. G. Lim and D. Zhi. Pricing options using implied trees: Evidence from ftse-100 options. Journal of Futures Markets, 22(7):601-626, 2002.

[32] D. B. Madan, P. P. Carr, and E. C. Chang. The variance gamma process and option pricing. European Finance Review, 2(1):79-105, 1998.

[33] R. C. Merton. Option pricing when underlying stock returns are discontinuous. Journal of Financial Economics, 3(1):125-144, 1976.

[34] A. Peiro. Skewness in financial returns. Journal of Banking $\mathcal{E}$ Finance, 23(6):847-862, 1999.

[35] S. T. Rachev, C. Menn, and F. J. Fabozzi. Fat-tailed and skewed asset return distributions: implications for risk management, portfolio selection, and option pricing, volume 139. John Wiley \& Sons, 2005.

[36] M. Rubinstein. Nonparametric tests of alternative option pricing models using all reported trades and quotes on the 30 most active cboe option classes from august 23 , 1976 through august 31, 1978. The Journal of Finance, 40(2):455-480, 1985.

[37] R. Schöbel and J. Zhu. Stochastic volatility with an ornstein-uhlenbeck process: an extension. European Finance Review, 3(1):23-46, 1999.

[38] L. O. Scott. Option pricing when the variance changes randomly: Theory, estimation, and an application. Journal of Financial and Quantitative analysis, 22(04):419-438, 1987. 
[39] S. E. Shreve. Stochastic calculus for finance II: Continuous-time models, volume 11. Springer Science \& Business Media, 2004.

[40] J. Shu and J. E. Zhang. Pricing s\&p 500 index options under stochastic volatility with the indirect inference method. Journal of Derivatives Accounting, 1(2):1-16, 2004.

[41] E. M. Stein and J. C. Stein. Stock price distributions with stochastic volatility: an analytic approach. Review of Financial Studies, 4(4):727-752, 1991.

[42] J. B. Wiggins. Option values under stochastic volatility: Theory and empirical estimates. Journal of Financial Economics, 19(2):351-372, 1987.

\section{Appendix A}

In Section 2.4, we set $A=\frac{\lambda_{1}}{4 \lambda_{2}}+\frac{1}{2 \sqrt{-k}}$ and $B=\frac{\lambda_{1}}{4 \lambda_{2}}-\frac{1}{2 \sqrt{-k}}$. Here we will prove that $A>0, B<0$.

1) $\lambda_{1}>0$. So we can get $B<0$ immediately. Now if we assume $A \leq 0$, then

$$
\frac{\lambda_{1}}{4 \lambda_{2}}+\frac{1}{2 \sqrt{-k}} \leq 0
$$

from which we can known

$$
\lambda_{1} \geq-\frac{2 \lambda_{2}}{\sqrt{-k}}>0
$$

Therefore, after some simplifications,

$$
0 \geq-2 \lambda_{2}(1-\alpha) \sigma^{2}
$$

which contradicts with the fact that $\lambda_{2}<0$. As a result, the assumption is not true and we have $A>0$. 
2) $\lambda_{1}<0$. Now we have $A>0$ from $\lambda_{1}<0$. If we assume $B \geq 0$, then

$$
\frac{\lambda_{1}}{4 \lambda_{2}}+\frac{1}{2 \sqrt{-k}} \geq 0,
$$

which implies

$$
-\lambda_{1} \geq-\frac{2 \lambda_{2}}{\sqrt{-k}}>0 .
$$

And then we can have

$$
\lambda_{1}^{2} \geq-\frac{4 \lambda_{2}^{2}}{k}>0,
$$

which again can be simplified to

$$
0 \geq-2 \lambda_{2}(1-\alpha) \sigma^{2} .
$$

As a result, Inequality $(A-2)$ contradicts with $\lambda_{2}<0$, which means that the assumption is incorrect and we obtain $B<0$.

Combining the situations we consider, we have $A>0, B<0$ for all possible values of $\lambda_{1}$. This has complete the proof.

\section{Appendix B}

According to the martingale pricing theory which tells us that $e^{-r t} U(S, v, t)$ should be a martingale when we seek the risk-neutral price, $U(S, v, t)$ should satisfy the following partial differential equation.

$$
\begin{aligned}
\frac{1}{2} v^{2 \alpha} S^{2} \frac{\partial^{2} U}{\partial S^{2}} & +\rho \sigma v S \frac{\partial^{2} U}{\partial v \partial S}+\frac{1}{2} \sigma^{2} v^{2-2 \alpha} \frac{\partial^{2} U}{\partial v^{2}}+r S \frac{\partial U}{\partial S}, \\
& +\left[\frac{1}{2}(1-\alpha) \sigma^{2} v^{1-2 \alpha}+\lambda_{1} v^{1-\alpha}+\lambda_{2} v\right] \frac{\partial U}{\partial v}-r U+\frac{\partial U}{\partial t}=0
\end{aligned}
$$


with the terminal and boundary conditions

$$
\begin{aligned}
U(S, v, T) & =\max (S-K, 0), \\
U(0, v, t) & =0 \\
\lim _{S \rightarrow \infty} \frac{U(S, v, t)}{S} & =1 .
\end{aligned}
$$

Now we assume the solution is of the form

$$
U(S, v, t)=S P_{1}-K e^{-r(T-t)} P_{2},
$$

which is exactly in the same form as that of the B-S formula. By substituting $(B-2)$ into the Equation $(B-1)$ along with the transform $x=\ln (S)$, we can obtain the partial differential equations that $P_{1}$ and $P_{2}$ must satisfy:

$$
\begin{aligned}
\frac{1}{2} v^{2 \alpha} \frac{\partial^{2} P_{j}}{\partial x^{2}} & +\rho \sigma v \frac{\partial^{2} P_{j}}{\partial v \partial x}+\frac{1}{2} \sigma^{2} v^{2-2 \alpha} \frac{\partial^{2} P_{j}}{\partial v^{2}}+\left(r+a_{j} v\right) \frac{\partial P_{j}}{\partial x} \\
& +\left[\frac{1}{2}(1-\alpha) \sigma^{2} v^{1-2 \alpha}+\lambda_{1} v^{1-\alpha}+\lambda_{2} v+b_{j} v\right] \frac{\partial P_{j}}{\partial v}+\frac{\partial P_{j}}{\partial t}=0
\end{aligned}
$$

where $j=\{1,2\}$ and the boundary condition becomes $P_{j}[x, v, t ; \ln (K)]=1_{\{x \geq \ln (K)\}}$. Actually, Equation $(B-3)$ can be transformed to two different dynamics of $x_{t}$ and $v_{t}$ with Feynman-Kac formula [39],

$$
\begin{aligned}
& d x=\left(r+a_{j} v\right) d t+v^{\alpha} d W_{t} \\
& d v=\left[\frac{1}{2}(1-\alpha) \sigma^{2} v^{1-2 \alpha}+\lambda_{1} v^{1-\alpha}+\lambda_{2} v+b_{j} v\right] d t+\sigma v^{1-\alpha} d B_{t}
\end{aligned}
$$

and thus it is not difficult to interpret that $P_{1}$ and $P_{2}$ represent the probability of exercising the option at maturity under different measures, respectively. Therefore, we can obtain

$$
\left.P_{j}[x, v, t ; \ln (K)]=P\left[x_{T} \geq \ln (K)\right] \mid x_{t}=x, v_{t}=v\right] .
$$


Let

$$
f_{j}(x, v, t ; \phi)=E\left[e^{i \phi x_{T}} \mid x_{t}=x, v_{t}=v\right],
$$

from which we can deduce that for any $s<t$,

$$
\begin{aligned}
E\left\{f_{j}(x, v, t ; \phi) \mid x_{s}, v_{s}\right\} & =E\left\{E\left[e^{i \phi x_{T}} \mid x_{s}, v_{s}\right] \mid x_{t}, v_{t}\right\} \\
& =E\left[e^{i \phi x_{T}} \mid x_{s}, v_{s}\right] \\
& =f_{j}(x, v, s ; \phi) .
\end{aligned}
$$

Thus, $f_{j}$ is a martingale, according to which we can obtain

$$
\begin{aligned}
\frac{1}{2} v^{2 \alpha} \frac{\partial^{2} f_{j}}{\partial x^{2}} & +\rho \sigma v \frac{\partial^{2} f_{j}}{\partial v \partial x}+\frac{1}{2} \sigma^{2} v^{2-2 \alpha} \frac{\partial^{2} f_{j}}{\partial v^{2}}+\left(r+a_{j} v\right) \frac{\partial f_{j}}{\partial x} \\
& +\left[\frac{1}{2}(1-\alpha) \sigma^{2} v^{1-2 \alpha}+\lambda_{1} v^{1-\alpha}+\lambda_{2} v+b_{j} v\right] \frac{\partial f_{j}}{\partial v}+\frac{\partial f_{j}}{\partial t}=0
\end{aligned}
$$

with the dynamics $(B-4)$ by applying the Itô lemma. On the other hand, from Equation $(B-6)$ we can easily work out the new terminal condition

$$
f_{j}[x, v, T ; \phi]=e^{i \phi x_{T}},
$$

by setting $t=T$.

Now we assume that the solution to the PDE $(B-7)$ takes the form of

$$
f_{j}=e^{C(T-t ; \phi)+D(T-t ; \phi) v^{2 \alpha}+E(T-t ; \phi) v^{\alpha}+i \phi x},
$$

and then substitute it into the PDE $(B-7)$. After some calculations and simplifications, the following equation needs to be satisfied

$$
\begin{aligned}
{\left[\frac{\partial D}{\partial t}\right.} & \left.+2 \alpha^{2} \sigma^{2} D^{2}+2 \alpha\left(i \phi \rho \sigma+b_{j}+\lambda_{2}\right) D+a_{j} i \phi-\frac{1}{2} \phi^{2}\right] v^{2 \alpha} \\
& +\left[\frac{\partial E}{\partial t}+\alpha\left(2 \alpha^{2} \sigma^{2} D+i \phi \rho \sigma+b_{j}+\lambda_{2}\right) E+2 \alpha \lambda_{1} D\right] v^{\alpha}
\end{aligned}
$$




$$
+\left[\frac{\partial C}{\partial t}+\frac{1}{2} \alpha^{2} \sigma^{2} E^{2}+\alpha^{2} \sigma^{2} D+\alpha \lambda_{1} E+r i \phi\right]=0
$$

With the fact that $v$ is an arbitrary variable, the satisfaction of Equation $(B-10)$ is equivalent to the satisfaction of the following three ordinary differential equations

$$
\begin{aligned}
& \frac{\partial D}{\partial \tau}=2 \alpha^{2} \sigma^{2} D^{2}+2 \alpha\left(i \phi \rho \sigma+b_{j}+\lambda_{2}\right) D+a_{j} i \phi-\frac{1}{2} \phi^{2}, \\
& \frac{\partial E}{\partial \tau}=\alpha\left(2 \alpha^{2} \sigma^{2} D+i \phi \rho \sigma+b_{j}+\lambda_{2}\right) E+2 \alpha \lambda_{1} D, \\
& \frac{\partial C}{\partial \tau}=\frac{1}{2} \alpha^{2} \sigma^{2} E^{2}+\alpha^{2} \sigma^{2} D+\alpha \lambda_{1} E+r i \phi,
\end{aligned}
$$

where $\mathrm{C}(0)=\mathrm{D}(0)=\mathrm{E}(0)=0$ and $\tau=T-t$.

Now if we let $A=2 \alpha^{2} \sigma^{2}, B=2 \alpha\left(i \phi \rho \sigma+b_{j}+\lambda_{2}\right), M=a_{j} i \phi-\frac{1}{2} \phi^{2},(B-11)$ can be further simplified to

$$
\frac{\partial D}{\partial \tau}=A D^{2}+B D+M
$$

which is exactly a Riccati Equation. By applying the transform $D=\frac{y^{\prime}}{-A y}$ to Equation $(B-14)$, we obtain

$$
\frac{d^{2} y}{d \tau^{2}}-B \frac{d y}{d \tau}+A M y=0
$$

with its corresponding boundary condition

$$
\left.\frac{d y}{d \tau}\right|_{\tau=0}=0
$$

The general solution of this newly derived ODE can be easily worked out as

$$
y=C_{1} e^{d^{+} \tau}+C_{2} e^{d^{-} \tau}
$$

where $d^{+}$and $d^{-}$are the two roots of the quadratic equation

$$
d^{2}-B d+A M=0,
$$


with

$$
d^{+}=\frac{B+\sqrt{B^{2}-4 A M}}{2}, \quad d^{-}=\frac{B-\sqrt{B^{2}-4 A M}}{2} .
$$

Furthermore, by making use of the boundary condition $(B-15)$, we can obtain $\frac{C_{1}}{C_{2}}=-\frac{d^{-}}{d^{+}}$. Therefore, $D(\tau ; \phi)$ can be finally worked out as

$$
\begin{aligned}
D(\tau ; \phi) & =-\frac{1}{A} \frac{C_{1} d^{+} e^{d^{+} \tau}+C_{2} d^{-} e^{d^{-} \tau}}{C_{1} e^{d^{+} \tau}+C_{2} e^{d^{-} \tau}}, \\
& =-\frac{1}{A} \frac{-d^{-} e^{d^{+} \tau}+d^{-} e^{d^{-} \tau}}{-\frac{d^{-}}{d^{+}} e^{d^{+} \tau}+e^{d^{-}}}, \\
& =\frac{d-2 \alpha\left(b_{j}+\lambda_{2}+i \phi \rho \sigma\right)}{4 \alpha^{2} \sigma^{2}}\left(\frac{1-e^{d \tau}}{1-g e^{d \tau}}\right) .
\end{aligned}
$$

After Equation $(B-11)$ is solved, we now turn to Equation $(B-12)$, which is nothing but a first-order linear non-homogeneous ODE with variable coefficients. To obtain a general solution, we set

$$
G_{1}(\tau)=2 \alpha^{2} \sigma^{2} D(\tau ; \phi)+\alpha b_{j}+\alpha \lambda_{2}+i \alpha \phi \rho \sigma, \quad G_{2}(\tau)=2 \alpha \lambda_{1} D(\tau ; \phi) .
$$

The solution to $(B-12)$ can then be derived as

$$
\begin{aligned}
E(\tau ; \phi) & =e^{\int_{0}^{\tau} G_{1}(t) d t} \int_{0}^{\tau} G_{2}(t) e^{-\int_{0}^{t} G_{1}(s) d s} d t \\
& =e^{p(\tau)} \int_{0}^{\tau} 2 \alpha \lambda_{1} D(t ; \phi) e^{-p(t)} d t .
\end{aligned}
$$

Since $D(\tau ; \phi)$ and $E(\tau ; \phi)$ are known by now, the derivation of $C(\tau ; \phi)$ is quite straightforward because it can be obtained simply by integrating on both sides of Equation $(B-13)$ and applying the boundary condition. This has completed the proof. 